\title{
Evaluating of a deep learning method for detecting exposed bars from images
}

P. Foucher ${ }^{1}$, G. Decor ${ }^{1,2}$, F. Bock ${ }^{1,3}$, P. Charbonnier ${ }^{1}$, F. Heitz ${ }^{2}$

${ }^{1}$ Cerema, research team ENDSUM; ${ }^{2}$ ICube, UMR 7357, University of Strasbourg, CNRS; ${ }^{3}$ Spacetec Gmbh

\section{Summary}

In this contribution, we use the deep learning Unet architecture for the segmentation of exposed bars from images of structure linings. The algorithm is trained using a set of images resized at different resolution. Images are divided into sub-images from which we randomly select patches, used as input data of the architecture. The impact of image resolution is evaluated. The generalisation capabilities of the learned model is finally assessed on three independent test image bases. The results show an F1 score performance of about $70 \%$ on two test databases. The third test image set gives a lower performance which is explained by a very different appearance of the exposed bars. This experiment illustrates the challenge of data distribution shift between training and test sets. It shows the interest of domain adaptation methods to address it. 


\section{NEAR SURFACE $\triangle$ GEOSCIENCE"}

Evaluating of a deep learning method for detecting exposed bars from images

\section{Introduction}

In this contribution, we propose and evaluate a learning-based algorithm for the segmentation (i.e., pixel-wise detection) of defects on engineering infrastructures, focusing on exposed reinforcing bars. Automatic detection of such defects is challenging due to the variability in appearance of both target objects and linings. In the infrastructure domain, machine learning methods are mainly focused on crack detection. There are some recent works dedicated to the recognition of exposed rebars (Mundt et al. 2019; Rubio et al. 2019). The main contribution of this paper is the systematic assessment of the segmentation. In particular, the impact of the image resolution on the performance of the algorithm is analysed. Moreover, we evaluate the capability of the model to generalize to test datasets.

\section{Segmentation method}

In this work, we are interested in a two-class segmentation of images: exposed bar pixels (positive category) and its opposite (negative category). The latter comprises healthy linings, other defects such as cracks and surrounding elements like sky, vegetation. To this end, we use the U-Net architecture (Ronneberger et al. 2015), a deep learning method algorithm dedicated to image segmentation. It is a two-part convolutional neural network that, first, encodes the input images into feature maps at multiple resolution levels using a set of convolutional filters and pooling functions. In the second half of the algorithm, low-resolution feature representations are decoded into grayscale images using upsampling operations. The output graylevel value corresponds to a confidence score, defined within the range $[0,1]$, in the classification of the pixel. A thresholding on these scores results in a binary image.
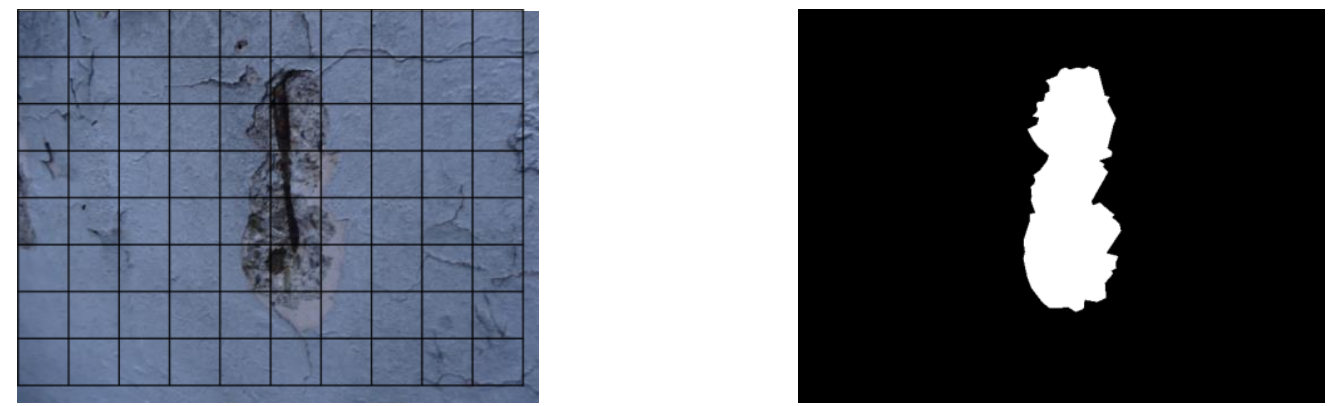

Figure 1 original image decomposed into a regular sub-image grid (left). Pixel-level ground truth of the image (right). White pixels belong to the "exposed bar" category.

Four different sets of images were annotated for training and testing the algorithm. The binary annotated images form the ground truth, as shown in Figure 1. Note that pixels of the spalled area around the exposed bar are labelled as positive. Details about the four datasets are given in Table 1. We use the CODEBRIM database from the research work of (Mundt et al., 2019) for which we completed the ground truth, initially built for image classification purposes, with a pixel-wise annotation. We also resized the images of the CODEBRIM dataset to $1536 \times 1152$ pixels. We collected the images of the other three datasets ourselves and generated the associated ground truths.

In the training process, the images are divided into sub-images of size $300 \times 300$ pixels according to a regular grid, as shown in Figure 1-left: $N_{\mathrm{s}}$ sub-images are selected to learn the segmentation model. The number and the choice of the selected sub-images depend on the resolution of the images. From every sub-image, 8 patches of size $256 \times 256$ pixels are randomly extracted. At every epoch of the training phase, data augmentation technics (Shorten and Khoshgoftaar 2019) are applied to each patch to integrate some variations: horizontal and vertical flip, random rotation, zoom and illumination changes. The use of data augmentation improves the learning performance and avoids model overfitting.

Our U-Net implementation is based on the architecture developed in (Drouyer, 2020), that includes a batch normalization process. We randomly divide our training dataset into $80 \%$ patches for training 


\section{NEAR SURFACE $\triangle$ GEOSCIENEE?}

itself and $20 \%$ patches to validate the model. Our algorithm is learned end-to-end, from scratch, with the ADAM optimizer and a batch size of 16. We initially planned 3000 epochs, but the learning performance does not change significantly after epoch 600 . The model that maximizes the F1-score (as defined in Equation 1) in the validation set is finally kept.

Table 1 Dataset composition. $N I_{P}$ is the number of images comprising at least one positive pixel. $N I_{T}$ is the total number of images in the dataset.

\begin{tabular}{|c|c|c|c|}
\hline Datasets & Training or test & $N I_{P} / N I_{T}$ & Size of images (in pixels) \\
\hline Pedestrian tunnel (West entrance) & Training & $119 / 128$ & $2448 \times 1836$ \\
\hline Pedestrian tunnel (East entrance) & Test & $49 / 78$ & $2448 \times 1836$ \\
\hline CODEBRIM & Test & $29 / 29$ & $1536 \times 1152$ \\
\hline Road tunnel & Test & $77 / 212$ & $1912 \times 1081$ \\
\hline
\end{tabular}

\section{Results and discussion}

In this section, we propose several series of experiments. As performance scores, we use Recall, Precision and F1-score metrics, as defined in Equation 1. TP (True Positive) is the number of wellclassified pixels as positive by the algorithm. FP (False Positive) and FN (False Negative) are the numbers of pixels wrongly classified by the algorithm as positive or negative respectively. The best performance is obtained when precision and recall tend simultaneously towards 1. F1-score measures a tradeoff between Recall et Precision.

$$
\text { Recall }=\frac{T P}{T P+F N} \quad ; \quad \text { Precision }=\frac{T P}{T P+F P} \quad ; \quad \text { F1_score }=2 \frac{\text { Recall.Precision }}{\text { Recall }+ \text { Precision }}
$$

The first experiment aims to analyse the impact of image resolution on the learning performance. Two learning sessions are performed with different sub-image sets extracted from original images of the west entrance of the pedestrian tunnel. In the first training (model 1), we combine $300 \times 300$ subimages extracted from full-resolution (i.e. $2448 \times 1836$ pixels) and half-resolution images (i.e. $1224 \times$ 918 pixels). The composition of the dataset is given in Table 2 . The second training (model 2) uses only sub-images extracted from half-resolution images, as seen in the last column of Table 2 . The half resolution of the original images obviously implies a smaller number of extracted sub-images. In the composition of sub-image sets, $50 \%$ of selected sub-images comprise at least $10 \%$ of positive pixels. It may be noticed that the numbers of pixels per category are imbalanced, as shown in Table 2 (last row). The best F1-scores obtained on the validation sets are $97.4 \%$ for model 1 and $98.2 \%$ for model 2 . The difference in the performance of these two trainings does not seem significant to us. We therefore keep these two models for further experimentation on the three test sets.

Table 2 Details on the training sets.

\begin{tabular}{|c|c|c|}
\hline & Model 1 & Model 2 \\
\hline Number of sub- images $\left(\mathrm{N}_{\mathrm{s}}\right)$ & 2089 & 947 \\
\hline Composition & $\begin{array}{c}\text { 1564 sub-images (from full-resolution) } \\
\text { 525 sub-images (from half-resolution) }\end{array}$ & $\begin{array}{c}\text { 947 sub-images (from } \\
\text { half-resolution) }\end{array}$ \\
\hline \% of positive pixels & $26 \%$ & $14 \%$ \\
\hline
\end{tabular}

The U-Net algorithm is applied to full-frame images, in a sliding-window manner at the size of patches $(256 \times 256$ pixels). We first assess the performance of model 1 and model 2 on test images of the pedestrian tunnel. Results are gathered in Table 3 for two image resolutions. In this experiment, there is no overlap between patches. For both learned models, we observe that the F1-scores are higher for the half-resolution test images compared to the full-resolution test images. Considering these halfresolution test images, the best result is obtained for model $2(\mathrm{~F} 1$-score $=0.669)$. Note that the difference between the two F1-scores is due to a significant improvement in the Precision score (0.598 in model 2 and 0.356 in model 1). Conversely, the Recall score is slightly lower for model 2 (0.76) than for model 1 (0.83). We thus keep model 2 for the last two experiments. 


\section{( AEAR SURFace - GEOSCIENGE'2}

Table 3 Results on images of pedestrian tunnel (East entrance) for a threshold of 0.5.

\begin{tabular}{|c|c|c|c|c|}
\hline Model & Test images & Precision & Recall & F1-score \\
\hline Training & Full-resolution images & 0.173 & 0.864 & 0.289 \\
\cline { 2 - 5 } 1 & Half-resolution images & 0.356 & 0.830 & 0.542 \\
\hline Training & Full-resolution images & 0.481 & 0.594 & 0.531 \\
\cline { 2 - 5 } 2 & Half-resolution images & 0.598 & 0.760 & 0.669 \\
\hline
\end{tabular}

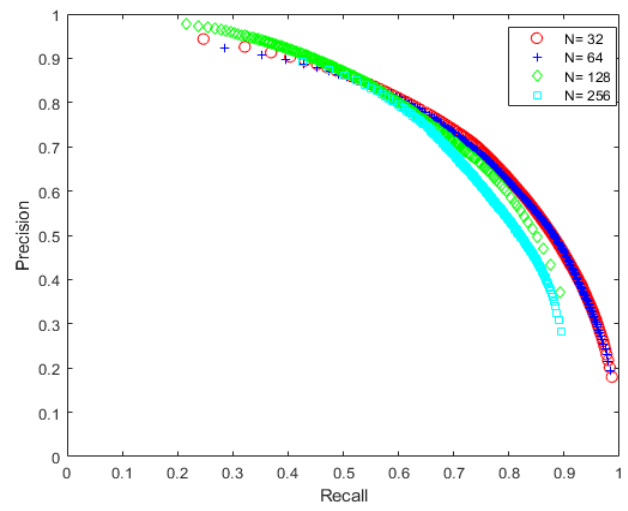

Table 4 Results on images of three datasets for a threshold of 0.5. $N=32$ pixels between patches.

\begin{tabular}{|c|c|c|c|}
\hline & Precision & Recall & F1-score \\
\hline Pedestrian Tunnel & 0.566 & 0.841 & 0.677 \\
\hline CODEBRIM & 0.728 & 0.716 & 0.722 \\
\hline Road Tunnel & 0.059 & 0.151 & 0.085 \\
\hline
\end{tabular}

Figure 2 Precision-recall curves for four levels of overlap.

We evaluate the performance of model 2 on test images of the pedestrian tunnel by applying an overlap between two successive patches. Figure 2 shows the Precision-Recall curves for different overlaps ( $N$ is the shift between two successive patches to be segmented). Remind that precision and recall curves are plotted by varying the binarization threshold on the result images. It may be seen that the area under the curves increases as $N$ decreases. We can therefore conclude that segmentation performance is improved when the overlap level increases.

In Figure 3, we first show two examples of segmentation from the test images of the pedestrian tunnel. The bars annotated in the ground truth seem to be well detected. In the second example, it may be observed that some false alarms are located on spalled areas that have no exposed bars.

To analyse the generalization capabilities of the model, we test the algorithm on images from the CODEBRIM and Road Tunnel databases. The results are shown in Table 4. The performance of the model measured on the CODEBRIM database $(\mathrm{F} 1$-score $=0.722)$ and the pedestrian tunnel database $(\mathrm{F} 1$-score $=0.677)$ are relatively close overall. However, compared to the performance on the pedestrian tunnel, we observe that the Recall decreases significantly (to 0.713 ), while Precision greatly improves (to 0.728). A result image of CODEBRIM dataset is shown in Figure 3. In this example, the object of interest and some false alarms along the bridge pier are detected.

An example of segmentation in road tunnel image is illustrated in Figure 3 (last row). The appearance of the exposed bars is here very different from the bars in the learned model. Without any processing, the recall is almost zero. By using negative images of the road tunnel, we obtain results of Table 4 (last row). Not surprisingly, the results are very poor. Too high a difference in the appearance of the objects to be recognized between the learned model and the test base prevents the detection of exposed bars. Domain adaptation methods can be explored for this issue.

\section{Conclusions}

In this work, we evaluated the performance of a U-Net architecture for the segmentation of exposed irons on images of structure linings. We analysed the impact of the resolution of the training images on the performance of the model. The lower the resolution, the better the performance. A possible explanation is that the context around the object of interest is better taken into account by the U-Net 


\section{( NEAR SURFACE GEOSCIENCE'21}

algorithm at low resolution than at high resolution. Assessment on independent test sets showed acceptable performance when the images and objects of interest were close to the learning domain. Otherwise, it decreases sharply.

In future experiments, we expect to introduce weighting into the parameters or functions of the learning algorithm to account for data imbalance. A multi-class segmentation could be also studied. A limitation of our model is the poor generalisation capabilities. Improving the ability to model exposed bars (and defects in general) can be achieved by diversifying the images used in training, but not only. It could be also necessary to experiment domain adaptation methods for this kind of application.

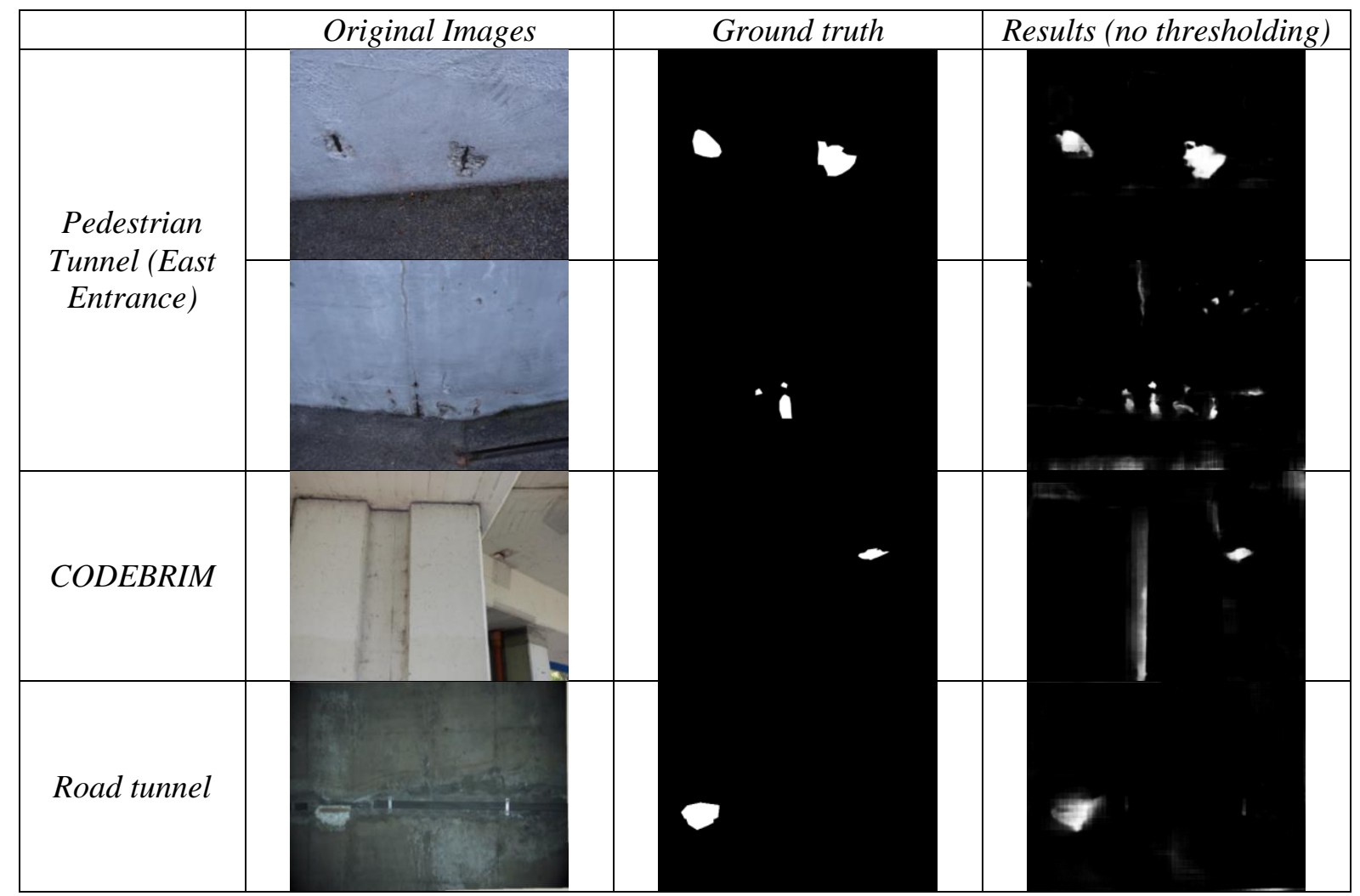

Figure 3 Sample images from the test datasets, ground truth and result.

\section{References}

Drouyer S. [2020] An "All Terrain" Crack Detector obtained by Deep Learning on Available Databases Image processing on line, 10, 105-122.

Mundt M., Majumder S., Murali S., Panetsos P. and Ramesh V. [2019] Meta-Learning Convolutional Neural Architectures for Multi-Target Concrete Defect Classification With the COncrete DEfect BRidge IMage Dataset. CVPR Conference, 11196-11205.

Ronneberger O., Fischer P. and Brox T. [2015] U-Net: Convolutional Networks for Biomedical Image Segmentation. Medical Image Computing and Computer-Assisted Intervention (MICCAI), 234-241.

Rubio J.J., Kashiwa T., Laiteerapong T., Deng W., Nagai K., Escalera S., Nakayama K., Matsuo Y. and Prendinger H. [2019] Multi-class structural damage segmentation using fully convolutional networks. Computers in Industry, 112, 103-121.

Shorten C. and Khoshgoftaar T.M. [2019] A survey on image data augmentation for deep learning. Journal of big data, 6(60). https://doi.org/10.1186/s40537-019-0197-0 